\title{
Dynamic Modeling and Chaotic Analysis of Gear Transmission System in a Braiding Machine with or without Random Perturbation
}

\author{
Zhang Yujing, Meng Zhuo, and Sun Yize \\ College of Mechanical Engineering, Donghua University, Shanghai 201620, China \\ Correspondence should be addressed to Meng Zhuo; mz@dhu.edu.cn
}

Received 1 September 2015; Revised 10 December 2015; Accepted 14 December 2015

Academic Editor: Evgeny Petrov

Copyright (C) 2016 Zhang Yujing et al. This is an open access article distributed under the Creative Commons Attribution License, which permits unrestricted use, distribution, and reproduction in any medium, provided the original work is properly cited.

\begin{abstract}
This paper is aimed at analyzing the dynamic behavior of the gear transmission system in a braiding machine. In order to observe the nonlinear phenomenon and reveal the time-varying gear meshing mechanism, a mathematical model with five degrees-offreedom gear system under internal and external random disturbance of gear system is established. With this model, bifurcation diagrams, Poincare maps, phase diagrams, power spectrum, time-process diagrams, and Lyapunov exponents are used to identify the chaotic status. Meanwhile, by these analytical methods, spur gear pair with or without random perturbation are compared. The numerical results suggest that the vibration behavior of the model is consistent with that of Clifford system. The chaotic system associated parameters are picked out, which can be helpful to the design and control of braiding machines.
\end{abstract}

\section{Introduction}

Regardless of the different braiding methods, the performance of the same braiding machine type primarily depends on the motion system.

In [1], the motion system of braiding machine is briefly introduced. Figure 1 is a detailed view of a large horizontal braiding machine. The yarn material is wound on bobbins, which are mounted on carriers. Every carrier follows the specific track by the guide chunk as their feet, moving forwards or backwards. Every gear is mounted on a shaft. At the same time, a horn disc with several cuts is fixed on the other side of the shaft. Horn disc pushes carrier base from one horn disc to another. Thus, carriers rotate toward the center, while the shafts rotate toward themselves. Obviously, one crucial thing of the motion system is the gear. Analyzing the gear meshing to keep down the vibration is an effective way of improving the performance of the braiding machine.

Nonlinear dynamics of gear system has been studied for many years. Blankenship and Kahraman [2] discussed the single-degree-of-freedom gear system with the superharmonic spur by taking parametric and clearance into account with the HBM method. Kahraman and Singh [3, 4] investigated the time-varying mesh stiffness and clearance nonlinearities in a geared system. Chang-Jian and Chang [5] studied the dynamic behavior of a single-degree-of-freedom spur gear system, revealing that the system exhibits periodic and chaotic vibration. Raghothama and Narayanan [6] have studied the three degrees-of-freedom nonlinear dynamic gear system by using bifurcation diagrams. Wang et al. [7] identified the parameters of gear system with sliding friction. Wang et al. and Xiaolin $[8,9]$ researched the three degrees-offreedom gear system under the external random disturbance. But with using the methods of three degrees-of-freedom gear system and ignoring the influence of internal incentive in the modelling of braiding machines, the results cannot be satisfactory.

During braiding, there are many spur sources to gears. The reactive force fluctuates severely. Not only external random disturbances must be considered, the internal disturbances cannot be ignored. On one hand, the yarn tension changes, especially when yarns are getting stuck. On the other hand, carriers change and the push force changes, which aggravates the fluctuation of forces. Moreover, as hundreds of gears participating in transmission, the influence of manufacture error and assembly error ought to be considered. 


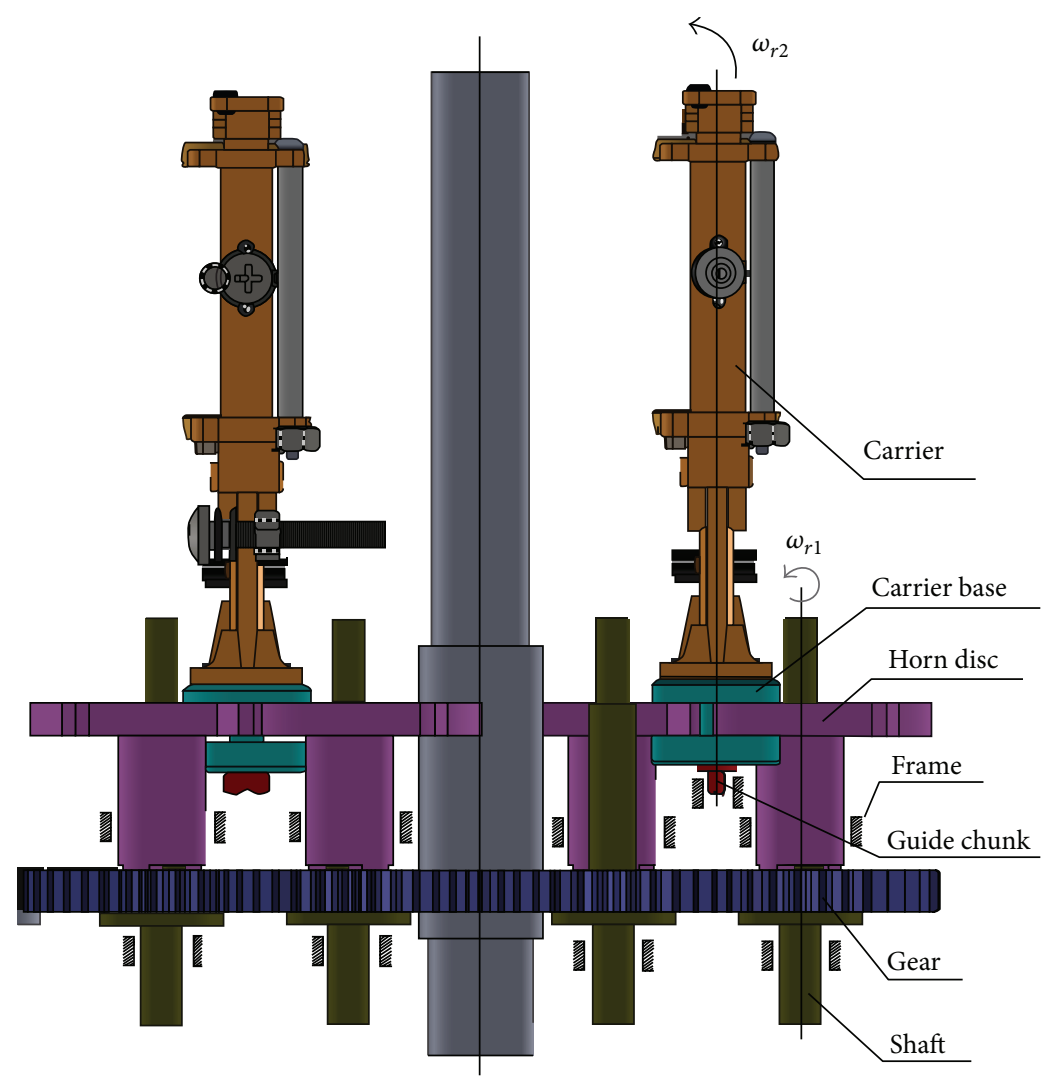

FIGURE 1: Motion system of the braiding machine.

Therefore, a study that focuses on analyzing the effects of both tangential and normal displacement, the eccentric force due to the manufacturing error, and assembly error to gear meshing is urgently needed.

\section{Dynamic Modeling}

To get the dynamic model, a schematic illustration is shown in Figure 2. Here, the influence from two directions displacement to the stiffness coefficient of bearings is considered. $m_{g 1}$, $m_{g 2}$ are the mass of gear; $m_{e}$ is effective mass; $I_{g 1}, I_{g 2}$ are the moment of inertia; $r_{g 1}, r_{g 2}$ are the base circle radius of gear; $y_{g 1}, y_{g 2}, x_{g 1}$, and $x_{g 2}$ are the center displacement of gear; $F_{b 1 x}, F_{b 1 y}, F_{b 2 x}$, and $F_{b 2 y}$ are the force transmitted from bearing; $c_{h}$ is the damping coefficient of gear mesh; $c_{b 1 x}, c_{b 1 y}$, $c_{b 2 x}$, and $c_{b 2 y}$ are the damping coefficient of bearing; $F_{e 1}, F_{e 2}$ are the eccentric force; $\varphi_{g 1}, \varphi_{g 2}$ are the angular displacement of gear; $\phi_{g 1}(\tau), \phi_{g 2}(\tau)$ are the phase angle of eccentric force; $f_{b 1 x}, f_{b 1 y}, f_{b 2 x}$, and $f_{b 2 y}$ are the displacement function of bearing; $e(\tau)$ is the static transmission error between gears; $k_{b 1 x}, k_{b 1 y}, k_{b 2 x}$, and $k_{b 2 y}$ are the stiffness coefficient of bearing; $k_{h}(\tau)$ is the time-varying mesh stiffness coefficient; $F_{0}$ is the mean force of input torque; $F_{v}$ is the variable amplitude of input torque; $\delta_{\Delta}$ is the random disturbance of relevant parameter (subscript $\Delta$ stands for parameter); $\rho_{g 1,2}$ is the mass eccentric arm; $\omega_{e 1}, \omega_{e 2}$ are the angular velocity of gear.
According to Newton's laws of motion, the differential equations of the gear system dynamics can be deduced as

$$
\begin{aligned}
& m_{g 1} \ddot{y}_{g 1}+\left(c_{b 1 y}+\delta_{c_{b 1 y}}\right) \dot{y}_{g 1} \\
& \quad+\left(c_{h}+\delta_{c_{h}}\right)\left[\dot{z}-\dot{y}_{g 1}+\dot{y}_{g 2}-\dot{e}(\tau)\right] \\
& \quad+k_{b 1 y} f_{b 1 y}\left(y_{g 1}\right)+k_{h}(\tau) f_{h}\left[z-y_{g 1}+y_{g 2}-e(\tau)\right] \\
& \quad+F_{b 1 y}+F_{e 1} \sin \left[\phi_{g 1}(\tau)\right]=0, \\
& m_{g 1} \ddot{x}_{g 1}+\left(c_{b 1 x}+\delta_{c_{b 1 x}}\right) \dot{x}_{g 1}+k_{b 1 x} f_{b 1 x}\left(x_{g 1}\right)+F_{b 1 x} \\
& \quad+F_{e 1} \cos \left[\phi_{g 1}(\tau)\right]=0, \\
& m_{g 2} \ddot{y}_{g 2}+\left(c_{b 2 y}+\delta_{c_{b 2 y}}\right) \dot{y}_{g 2} \\
& \quad-\left(c_{h}+\delta_{c_{h}}\right)\left[\dot{z}-\dot{y}_{g 1}+\dot{y}_{g 2}-\dot{e}(\tau)\right] \\
& +k_{b 2 y} f_{b 2 y}\left(y_{g 2}\right)-k_{h}(\tau) f_{h}\left[z-y_{g 1}+y_{g 2}-e(\tau)\right] \\
& +F_{b 2 y}+F_{e 2} \sin \left[\phi_{g 2}(\tau)\right]=0, \\
& m_{g 2} \ddot{x}_{g 2}+\left(c_{b 2 x}+\delta_{c_{b 2 x}}\right) \dot{x}_{g 2}+k_{b 2 x} f_{b 2 x}\left(x_{g 2}\right)+F_{b 2 x} \\
& +F_{e 2} \cos \left[\phi_{g 2}(\tau)\right]=0, \\
& m_{e} \ddot{z}+\left(c_{h}+\delta_{c_{h}}\right)\left[\dot{z}-\dot{y}_{g 1}+\dot{y}_{g 2}-\dot{e}(\tau)\right]
\end{aligned}
$$




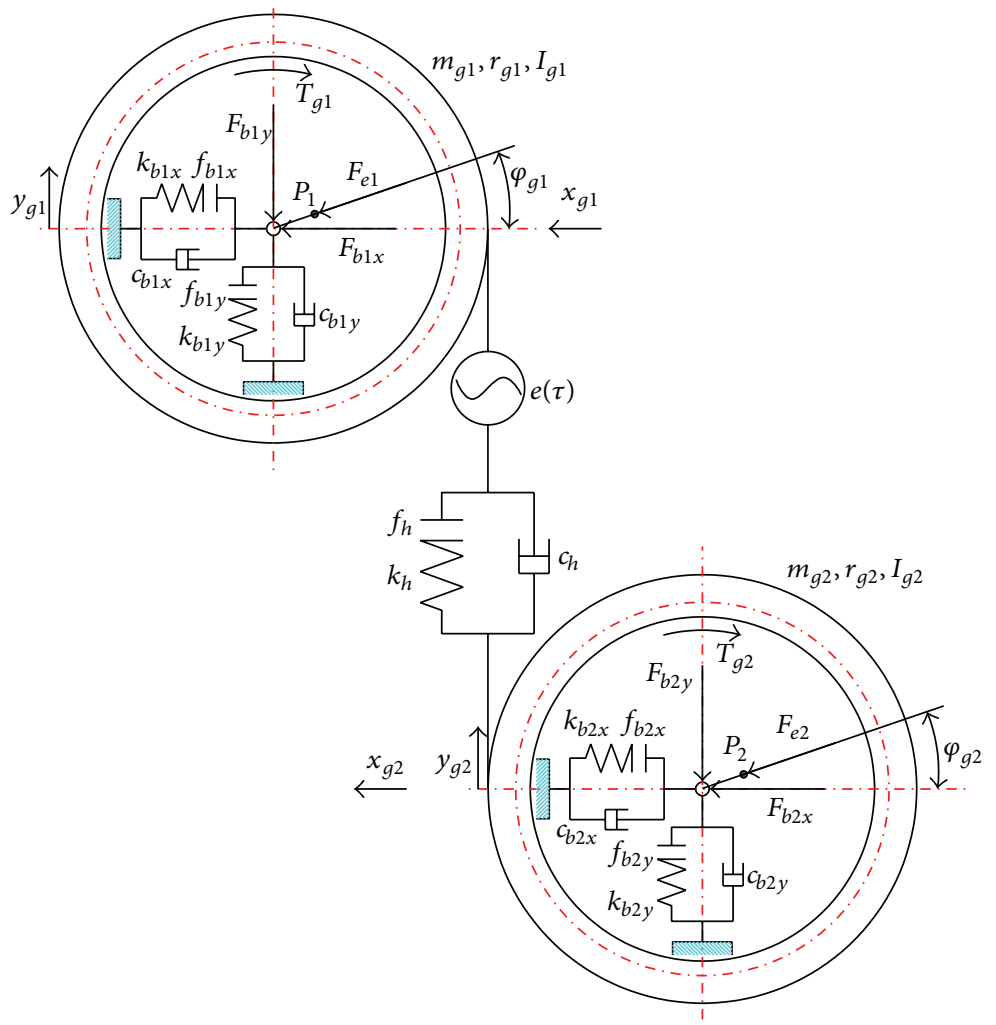

Figure 2: Dynamic model of gear system.

$$
\begin{aligned}
& +k_{h}(\tau) f_{h}\left[z-y_{g 1}+y_{g 2}-e(\tau)\right]-F_{0}-F_{v}(\tau) \\
& -\delta_{F}=0
\end{aligned}
$$

where

$$
\begin{aligned}
& z=r_{g 1}\left(\varphi_{g 1}-\delta_{\varphi_{g 1}}\right)-r_{g 2}\left(\varphi_{g 2}-\delta_{\varphi_{g 2}}\right), \\
& m_{e}=\frac{I_{g 1} I_{g 2}}{\left(I_{g 1} r_{g 2}^{2}+I_{g 2} r_{g 1}^{2}\right)}, \\
& f_{b 1 x}(u), f_{b 1 y}(u), f_{b 2 x}(u), f_{b 2 y}(u), f_{h}(u) \\
& = \begin{cases}u-b, & u>b \\
0, & -b \leq u \leq b \\
u+b, & u<-b\end{cases} \\
& u=y_{g 1}, y_{g 2}, x_{g 1}, x_{g 2}, \alpha, \\
& F_{e 1}=m_{g 1} \rho_{g 1} \omega_{e 1}^{2}, \\
& F_{e 2}=m_{g 2} \rho_{g 2} \omega_{e 2}^{2} \text {. }
\end{aligned}
$$

Meanwhile, $k_{h}(\tau)$ and $e(\tau)$ are time-varying functions. They can be expanded into the following equations by Fourier transform:

$$
\begin{aligned}
k_{h}(\tau) & =k_{0}+\sum_{i=1}^{\infty} k_{v} \cos \left[i\left(\omega_{h}+\delta_{\omega_{h}}\right) \tau+\phi_{h i}\right], \\
e(\tau) & =e_{v} \sum_{j=1}^{\infty} \sin \left(j \omega_{h} \tau+\phi_{h j}\right),
\end{aligned}
$$

where $k_{0}$ is the mean mesh stiffness; $k_{v}$ is the variable amplitude of mesh stiffness; $e_{v}$ is the amplitude of transmission error; $\omega_{h}$ is the driving frequency; $\phi_{h i}, \phi_{h j}$ are the phase angle of each harmonic term.

As is known, fundamental frequency influences the model most. Therefore, neglecting the influence of phase angle, $k_{h}(\tau)$ and $e(\tau)$ can be written as

$$
\begin{aligned}
k_{h}(\tau) & =k_{0}+k_{v} \cos \left[\left(\omega_{h}+\delta_{\omega_{h}}\right) \tau\right], \\
e(\tau) & =e_{v} \sin \left(\omega_{h} \tau\right) .
\end{aligned}
$$

For convenience and to simplify the equations, the following parameters are defined:

$$
\begin{aligned}
\alpha= & r_{g 1}\left(\varphi_{g 1}-\delta_{\varphi_{g 1}}\right)-r_{g 2}\left(\varphi_{g 2}-\delta_{\varphi_{g 2}}\right)-y_{g 1} \\
& +y_{g 2}-e(\tau) \\
t= & \omega_{n} \tau \\
\omega_{n}= & \sqrt{\frac{k_{0}}{m_{e}}}
\end{aligned}
$$




$$
\begin{aligned}
& \omega_{1}=\sqrt{\frac{k_{b 1} y}{m_{g 1}}}, \\
& \omega_{2}=\sqrt{\frac{k_{b 2 y}}{m_{g 2}}}, \\
& b^{*}=\frac{b}{b_{c}}, \\
& y_{g 1}^{*}=\frac{y_{g 1}}{b_{c}} \text {, } \\
& y_{g 2}^{*}=\frac{y_{g 2}}{b_{c}}, \\
& \alpha^{*}=\frac{\alpha}{b_{c}}, \\
& k_{b 1 x, y}^{*}=\frac{k_{b 1 x, y}}{b_{c}} \text {, } \\
& k_{b 2 x, y}^{*}=\frac{k_{b 2 x, y}}{b_{c}} \text {, } \\
& k_{0}^{*}=\frac{k_{0}}{b_{c}}, \\
& k_{v}^{*}=\frac{k_{v}}{b_{c}}, \\
& \omega_{h}^{*}=\frac{\omega_{h}}{\omega_{n}}, \\
& \omega_{1}^{*}=\frac{\omega_{1}}{\omega_{n}}, \\
& \omega_{2}^{*}=\frac{\omega_{2}}{\omega_{n}}, \\
& \varepsilon=\frac{k_{h v}}{k_{h 0}},
\end{aligned}
$$

where $\alpha$ is the relative torsional displacement of gear system; $\omega_{1}, \omega_{2}$ are equivalent frequency; $b$ is half of total gear backlash; $b_{c}$ is characteristic length of gear backlash; $\Delta^{*}$ is dimensionless parameter (subscript $\Delta$ stands for parameter except for coefficient of damping and stiffness).

To analyze the equations, let $x_{1}=y_{g 1}^{*}, x_{2}=y_{g 1}^{*}, x_{3}=x_{g 1}^{*}$, $x_{4}=x_{g 1}^{*}, x_{5}=y_{g 2}^{*}, x_{6}=y_{g 2}^{*}, x_{7}=x_{g 2}^{*}, x_{8}=x_{g 2}^{*}, x_{9}=\alpha^{*}$, and $x_{10}=\alpha^{*}$, assign $b=b_{c}$, and neglect initial phase angle of $\phi_{g 1}, \phi_{g 2}$. By using the defined dimensionless parameters above, the state equations (1) of the system can be turned into

$$
\begin{aligned}
\dot{x}_{1}= & x_{2}, \\
\dot{x}_{2}= & -2\left(\gamma_{11}+\delta_{\gamma_{11}}\right) x_{2}-2\left(\gamma_{15}+\delta_{\gamma_{15}}\right) x_{10} \\
& -\eta_{11} f_{b 1 y}\left(x_{1}\right)-\eta_{15}(t) f_{h}\left(x_{9}\right)-F_{b 1 y}^{*} \\
& -\frac{\rho_{g 1}^{*}\left(\omega_{h}^{*}+\delta_{\omega_{h}^{*}}\right)^{2}}{z_{1}^{2} \cos \left[\left(\omega_{h}^{*}+\delta_{\omega_{h}^{*}}\right) t / z_{1}\right]}
\end{aligned}
$$

$$
\begin{aligned}
& \dot{x}_{3}=x_{4} \text {, } \\
& \dot{x}_{4}=-2\left(\gamma_{22}+\delta_{\gamma_{22}}\right) x_{4}-\eta_{22} f_{b 1 x}\left(x_{3}\right)-F_{b 1 x}^{*} \\
& -\frac{\rho_{g 1}^{*}\left(\omega_{h}^{*}+\delta_{\omega_{h}^{*}}\right)^{2}}{z_{1}^{2} \sin \left[\left(\omega_{h}^{*}+\delta_{\omega_{h}^{*}}\right) t / z_{1}\right]}, \\
& \dot{x}_{5}=x_{6} \text {, } \\
& \dot{x}_{6}=-2\left(\gamma_{33}+\delta_{\gamma_{33}}\right) x_{6}+2\left(\gamma_{35}+\delta_{\gamma_{35}}\right) x_{10} \\
& -\eta_{33} f_{b 2}\left(x_{5}\right)+\eta_{35}(t) f_{h}\left(x_{9}\right)-F_{b 2 y}^{*} \\
& -\frac{\rho_{g 2}^{*}\left(\omega_{h}^{*}+\delta_{\omega_{h}^{*}}\right)^{2}}{z_{2}^{2} \cos \left[\left(\omega_{h}^{*}+\delta_{\omega_{h}^{*}}\right) t / z_{2}\right]}, \\
& \dot{x}_{7}=x_{8} \text {, } \\
& \dot{x}_{8}=-2\left(\gamma_{44}+\delta_{\gamma_{44}}\right) x_{8}-\eta_{44} f_{b 2 x}\left(x_{7}\right)-F_{b 2 x}^{*} \\
& -\frac{\rho_{g 2}^{*}\left(\omega_{h}^{*}+\delta_{\omega_{h}^{*}}\right)^{2}}{z_{2}^{2} \sin \left[\left(\omega_{h}^{*}+\delta_{\omega_{h}^{*}}\right) t / z_{2}\right]}, \\
& \dot{x}_{9}=x_{10} \text {, } \\
& \dot{x_{10}}=\dot{x_{2}}-\dot{x_{6}}-2\left(\gamma_{55}+\delta_{\gamma_{55}}\right) x_{10}-\eta_{55}(t) f_{h}\left(x_{9}\right) \\
& +F_{0}^{*}+e_{v}^{*}\left(\omega_{h}^{*}+\delta_{\omega_{h}^{*}}\right)^{2} \sin \left[\left(\omega_{h}^{*}+\delta_{\omega_{h}^{*}}\right) t\right] \\
& +\delta_{F}^{*}
\end{aligned}
$$

where $z_{1}, z_{2}$ are the number of teeth; $\gamma_{i j}$ is the dimensionless damping coefficient; $\eta_{i j}$ is the dimensionless stiffness coefficient $(i, j=1, \ldots, 5) ; F_{b 1}^{*}=F_{b 1} / m_{g 1} b_{c} \omega_{n}^{2} ; F_{b 2}^{*}=F_{b 2} / m_{g 2} b_{c} \omega_{n}^{2}$; $\gamma_{11}=c_{b 1 y} / 2 m_{g 1} \omega_{n} ; \gamma_{15}=c_{h} / 2 m_{g 1} \omega_{n} ; \gamma_{22}=c_{b 1 x} / 2 m_{g 1} \omega_{n}$; $\gamma_{33}=c_{b 2 y} / 2 m_{g 2} \omega_{n} ; \gamma_{35}=c_{h} / 2 m_{g 2} \omega_{n} ; \gamma_{44}=c_{b 2 x} / 2 m_{g 2} \omega_{n}$; $\gamma_{55}=c_{h} / 2 m_{e} \omega_{n} ; \eta_{11}=k_{b 1 y} / 2 m_{g 1} \omega_{n} ; \eta_{22}=k_{b 1 x} / 2 m_{g 1} \omega_{n} ;$ $\eta_{33}=k_{b 2 y} / 2 m_{g 2} \omega_{n} ; \eta_{44}=k_{b 2 x} / 2 m_{g 2} \omega_{n} ; \eta_{55}(t)=4 \eta_{15}(t)=$ $4 \eta_{35}(t)=1-\left(\varepsilon+\delta_{\varepsilon}\right) \cos \left[\left(\omega_{h}^{*}+\delta_{\omega_{h}^{*}}\right) t\right]$.

\section{Analysis of Numerical Simulation Results and Discussion}

Based on the mathematical model established above, the nonlinear dynamic equations were solved by differenceiteration method. During the numerical simulation, the time step was assigned a value of 0.05 , and, for convenience, let $\omega=\omega_{h}^{*}$. In the study, the $\omega$ is variable, while other parameters are constant: $m_{1}=m_{2}=m_{e}=0.456 \mathrm{~kg}, I_{g 1}=I_{g 2}=$ $6 \times 10^{-6} \mathrm{~kg} \cdot \mathrm{m}^{2}, z_{1}=z_{2}=30, b_{c}=0.068 \mathrm{~mm}, e_{v}=0.034 \mathrm{~mm}$, $k_{0}=2.23 \times 10^{8} \mathrm{~N} \cdot \mathrm{m}$, and $k_{v}=1.12 \times 10^{7} \mathrm{~N} \cdot \mathrm{m}$. Here, $k_{0}$, $k_{v}$ are obtained by reference to $[10,11]$. Therefore, the value of system parameters can be set as $e_{v}^{*}=0.05, F_{b 1 y}^{*}=0.2$, $F_{b 1 x}^{*}=0.2, F_{b 2 y}^{*}=-0.2, F_{b 2 x}^{*}=0.2, F_{0}^{*}=0.2, \eta_{11}=1.1$, $\eta_{22}=1.1, \eta_{33}=1.1, \eta_{44}=1.1, \varepsilon=0.18, \gamma_{11}=0.01$, $\gamma_{22}=0.01, \gamma_{33}=0.01, \gamma_{44}=0.01, \gamma_{15}=0.012, \gamma_{35}=0.012$, 


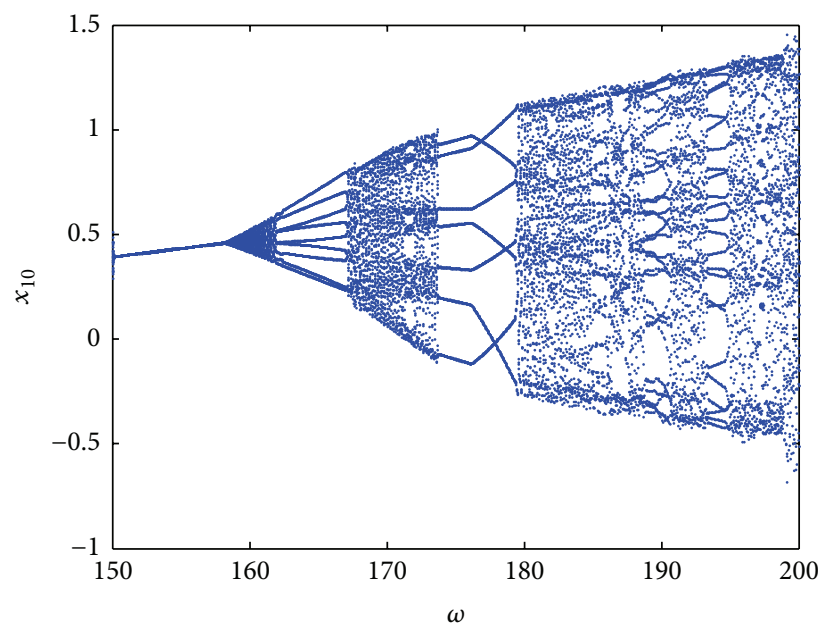

(a) Vibrational bifurcation diagram

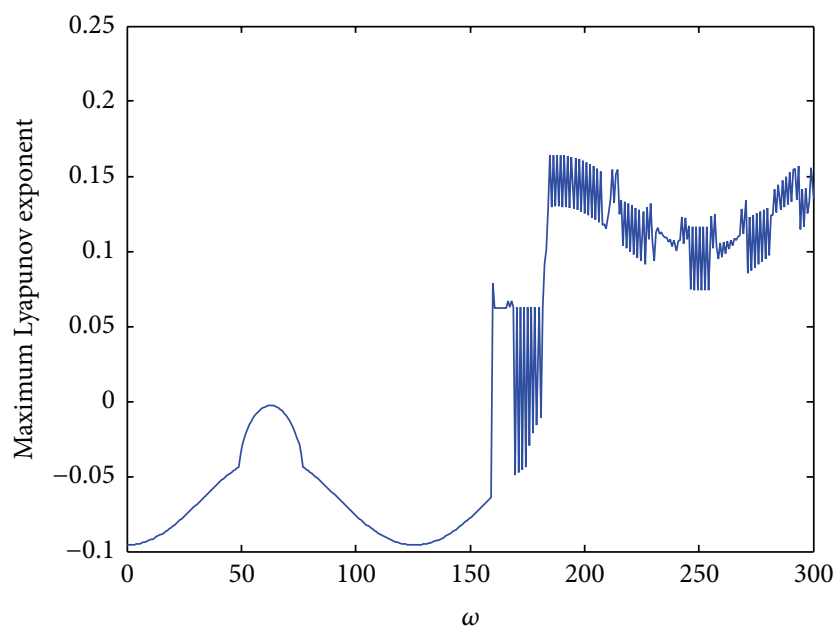

(b) Maximum Lyapunov exponent curve

FIGURE 3: System response without random perturbation.

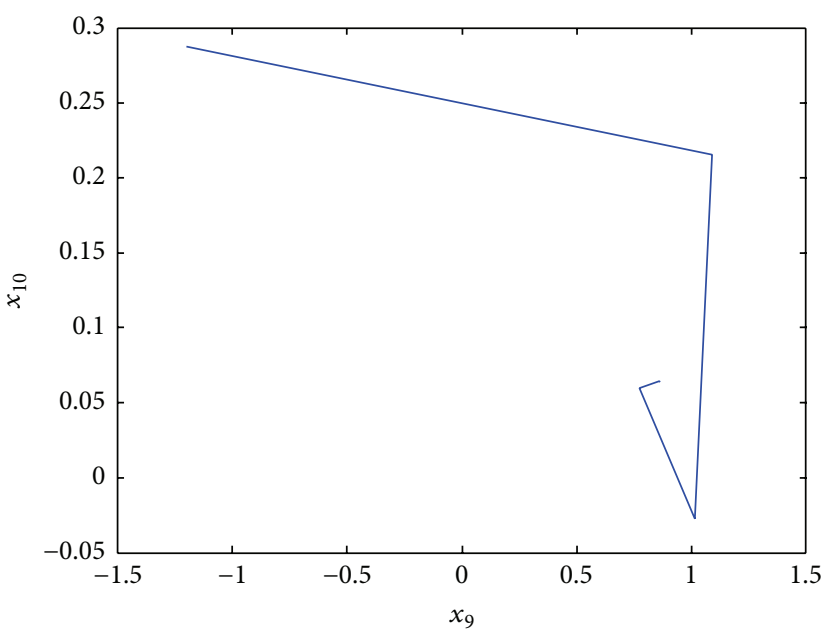

(a) Phase trajectories

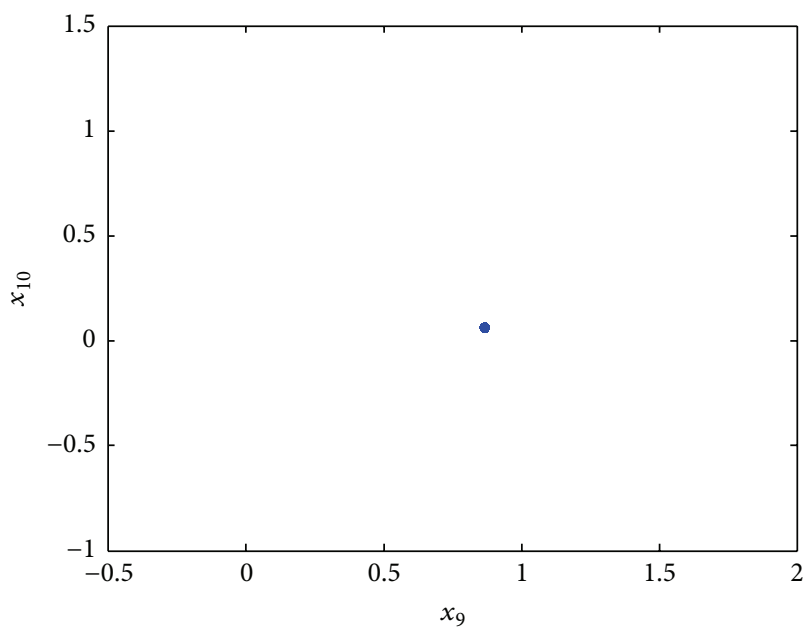

(b) Poincare maps

FIGURE 4: $\omega=110$ without random perturbation.

$\gamma_{55}=0.05, \rho_{g 1}^{*}=0.1$, and $\rho_{g 2}^{*}=0.1$. The initial state is set as $x_{1}(0)=0, x_{2}(0)=-0.1, x_{3}(0)=0, x_{4}(0)=-0.1$, $x_{5}(0)=0, x_{6}(0)=-0.1, x_{7}(0)=0, x_{8}(0)=-0.1, x_{9}(0)=0$, and $x_{10}(0)=-0.1$.

3.1. Analysis of System without Random Perturbation. The bifurcation diagram of system without random perturbation, which is generated with $\omega$ and $x_{10}$ in (10)-(15), and the maximum Lyapunov exponent curve graph are shown in Figure 3, while Poincare maps and corresponding phase trajectories are drawn in Figures 4-9.

On one hand, from the bifurcation diagram in Figure 3(a), it can be observed that the system presents a nonlinear vibration periodically. (1) When $\omega<153.5$, the system only has one periodic point, which is shown in Figure 3(a). From the phase trajectory of $\omega=110$ in Figure 4, it can be seen that iteration converges on one periodic point quickly, which means the motion of the nonlinear dynamic system is steady. (2) As the parameter $\omega$ increases from 153.70 to 174.18 , the number of periodic points varies. (3) Nonetheless, when $174.19<\omega<179.15$, there are 7 periodic points according to Figure 3(a). And a case of $\omega=175$ is shown in Figure 5. (4) During $179.16<\omega<180.05$, there are 18 periodic points in the diagram in Figure 3(a), which is a consequence of period bifurcations. And Figure 6 is an example of $\omega=180$. (5) In Figure 3(a), while $180.06<\omega<220$, the system exhibits a chaotic behavior. And Figure 7 is an illustration of $\omega=200$. (6) By observing Figures 3(a) and 8, when $220<\omega<261.17$, the periodic points form a limit-circle. (7) Until $\omega$ is above 261.18 in Figure 3(a), with amplitudes expanding, the vibration and noise increase rapidly. At last, in Figure 9, while $\omega=262$ system becomes divergent and uncontrollable.

On the other hand, from the maximum Lyapunov exponent curve graph in Figure 3(b), the nonlinear phenomenon also can be deduced. Here, the maximum 


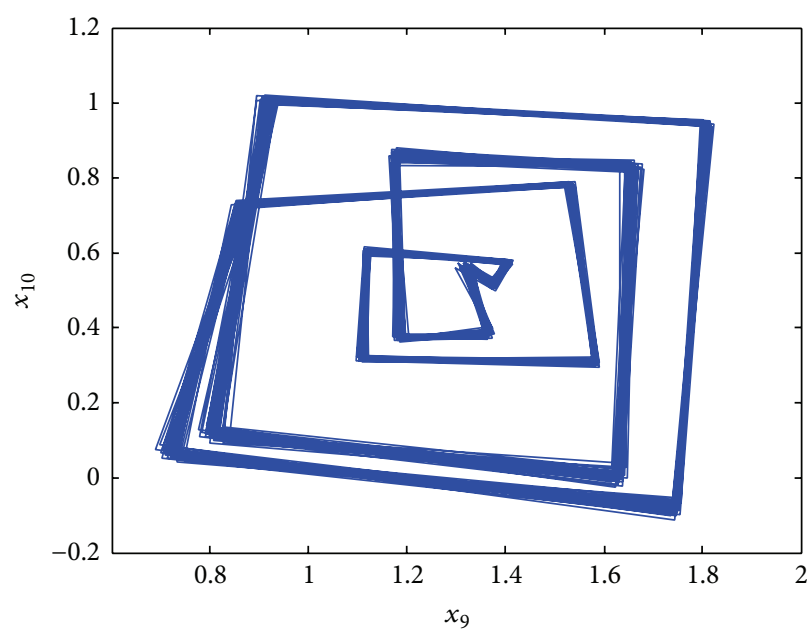

(a) Phase trajectories

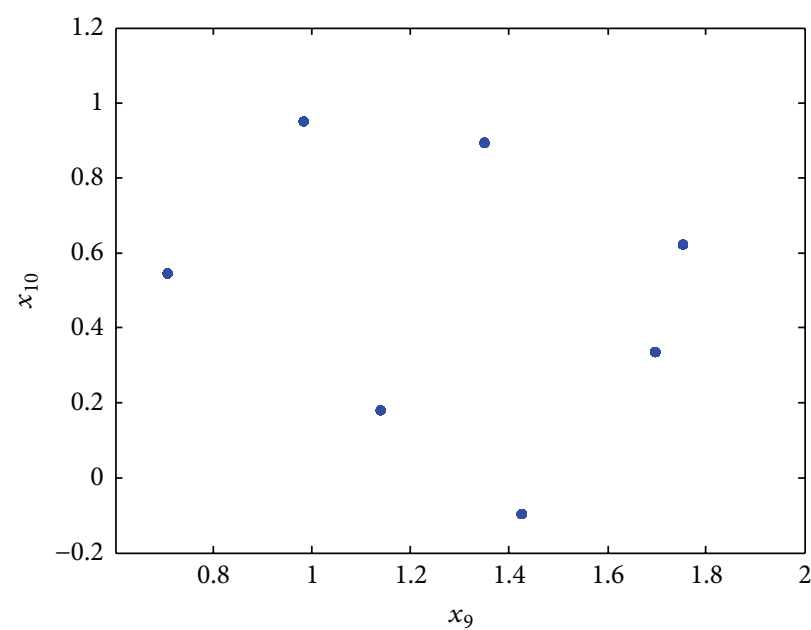

(b) Poincare maps

FIGURE 5: $\omega=175$ without random perturbation.

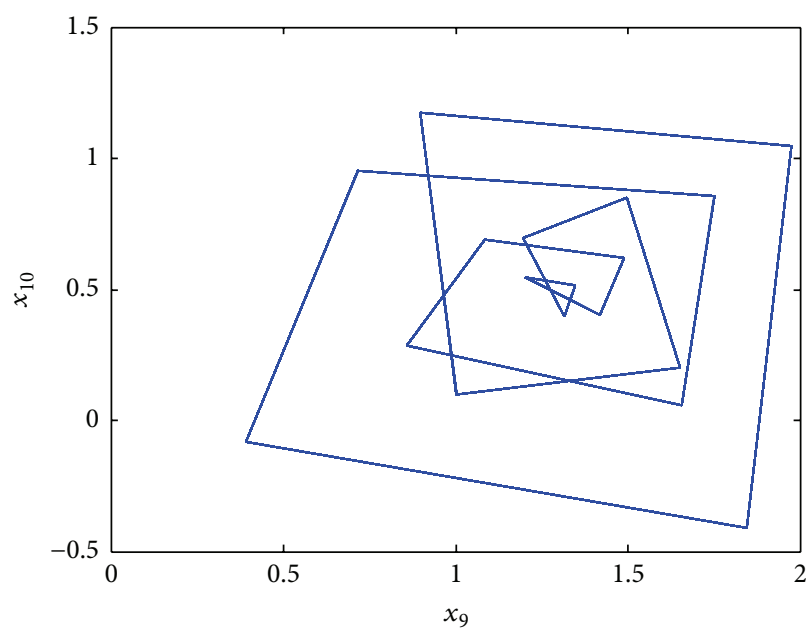

(a) Phase trajectories

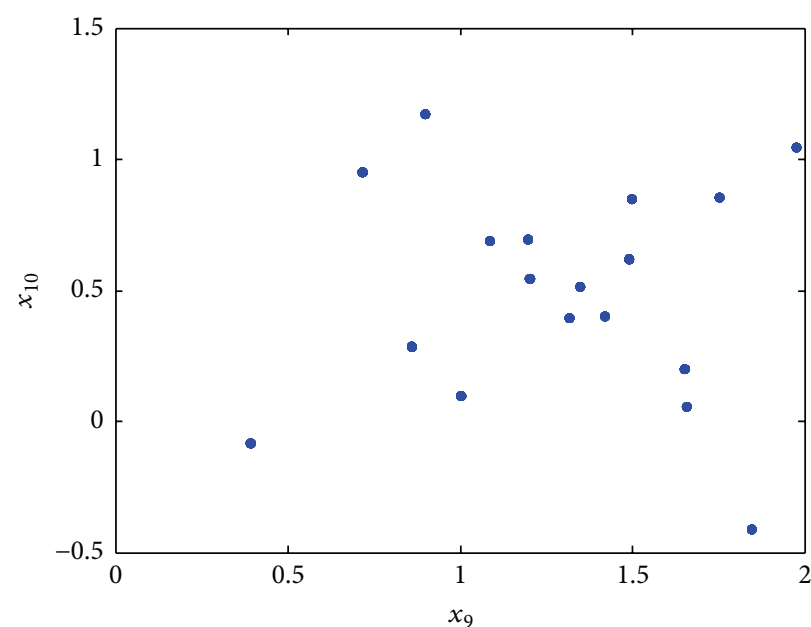

(b) Poincare maps

FIGURE 6: $\omega=180$ without random perturbation.

Lyapunov exponents are calculated by using Jacobi matrix. Meanwhile, Lyapunov exponent is defined as

$$
\begin{aligned}
& \lambda_{k}=\lim _{i \rightarrow \infty} \frac{1}{i} \ln \left|\lambda_{k}^{(i)}\right|, \\
& \quad k=1, \ldots, n \text { where }\left|\lambda_{1}^{i}\right| \geq\left|\lambda_{2}^{i}\right| \geq \cdots \geq\left|\lambda_{n}^{i}\right| .
\end{aligned}
$$

When $\omega$ is assigned about 160 to 180, the maximum Lyapunov exponents oscillate around zero. According to the L-S theory, system bifurcates, which is consistent with the bifurcation diagram. When $\omega$ keeps increasing, the maximum Lyapunov exponents always stay positive. Chaotic behavior occurs, and after that, system becomes diverging gradually.

3.2. Analysis of System with Random Perturbation. While observing system with random perturbation, the following parameters are assigned:

$$
\begin{aligned}
\delta_{\gamma_{11}}, \delta_{\gamma_{15}}, \delta_{\gamma_{22}}, \delta_{\gamma_{33}}, \delta_{\gamma_{35}}, \delta_{\gamma_{44}}, \delta_{\gamma_{55}}, \delta_{\varepsilon}, \delta_{F}^{*} \\
\sim N\left(0,0.001^{2}\right), \\
\delta_{\omega_{n}^{*}} \sim N\left(0,0.00005^{2}\right) .
\end{aligned}
$$

Meanwhile, values of other parameters are the same as in Section 3.1.

The Poincare maps and corresponding phase trajectories are drawn from Figures 10-15. It can be inferred that, after taking random perturbation into consideration, the system diverges in different degree. In Figures 10 and 11, the system still keeps stable. Yet, while $\omega=180$, the system advanced exhibits a chaotic behavior in Figure 12. By comparison, the system without random perturbation only has 18 periodic points.

To obtain more detail of the system under random perturbation, power spectrum, time-process diagrams, and 


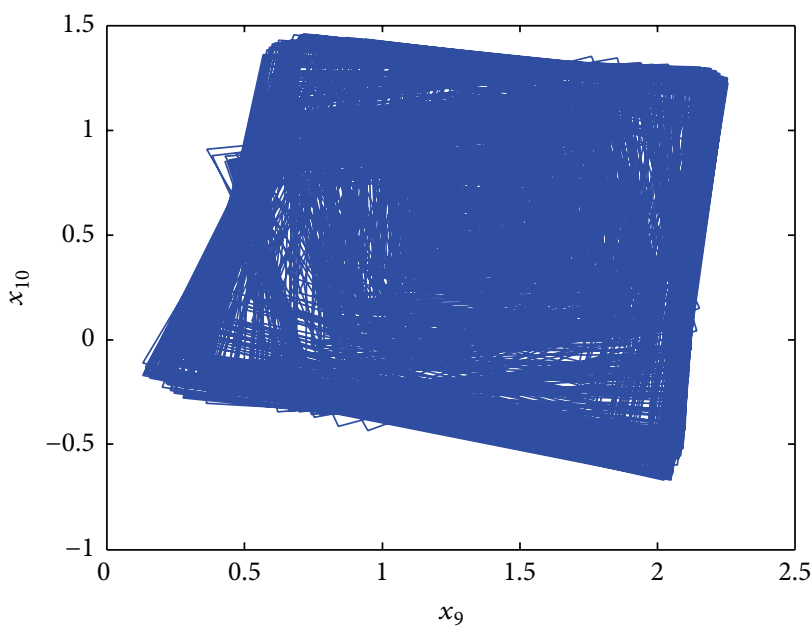

(a) Phase trajectories

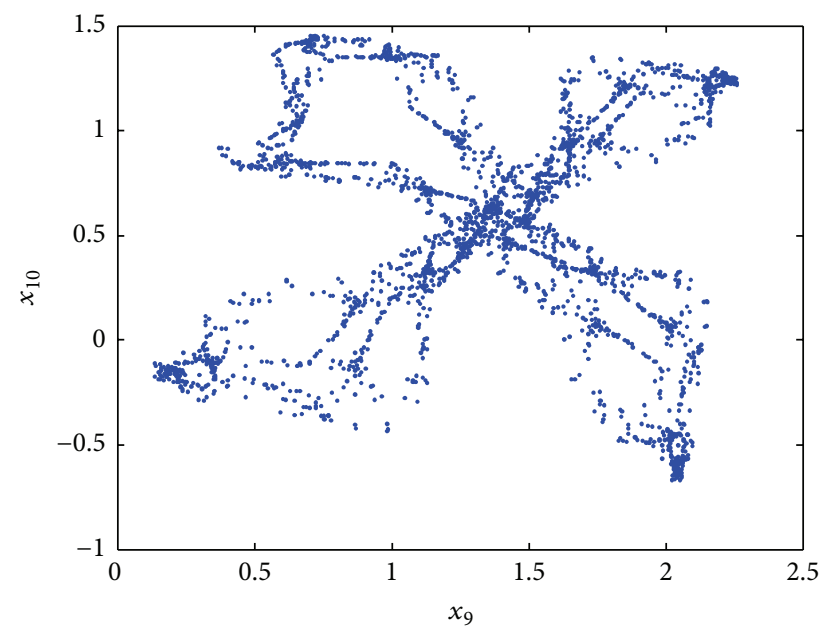

(b) Poincare maps

FIGURE 7: $\omega=200$ without random perturbation.

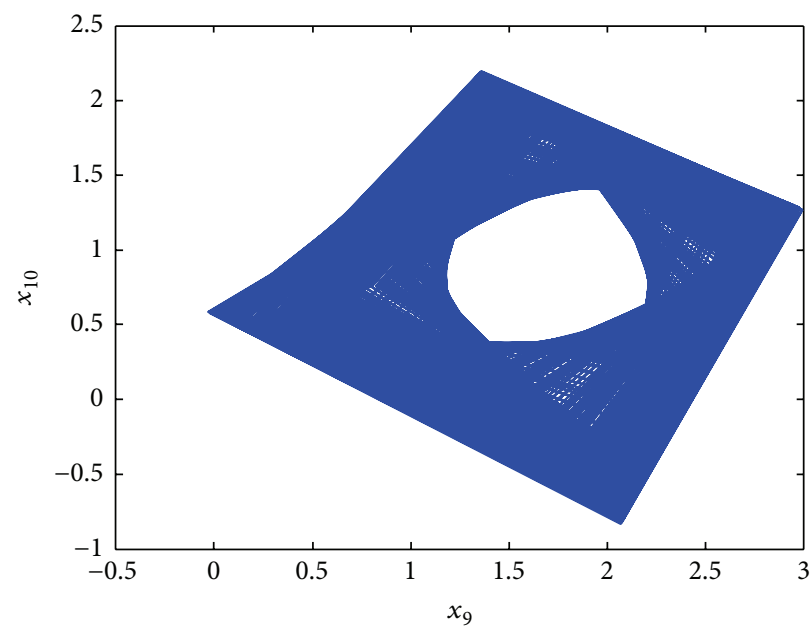

(a) Phase trajectories

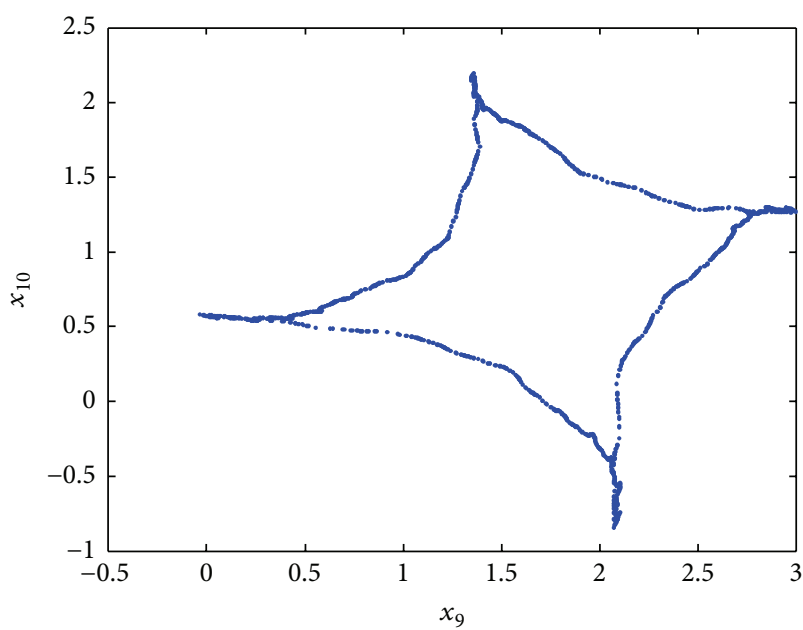

(b) Poincare maps

Figure 8: $\omega=240$ without random perturbation.

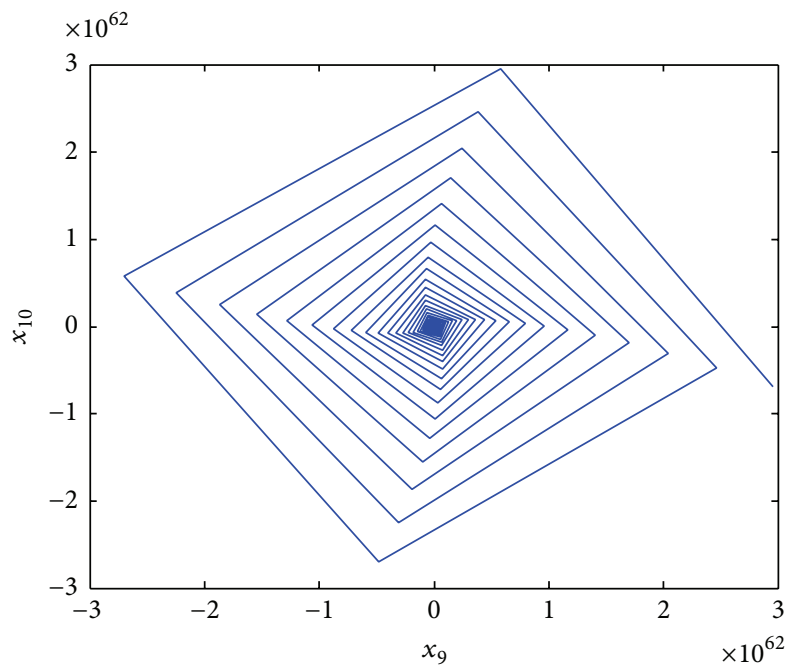

(a) Phase trajectories

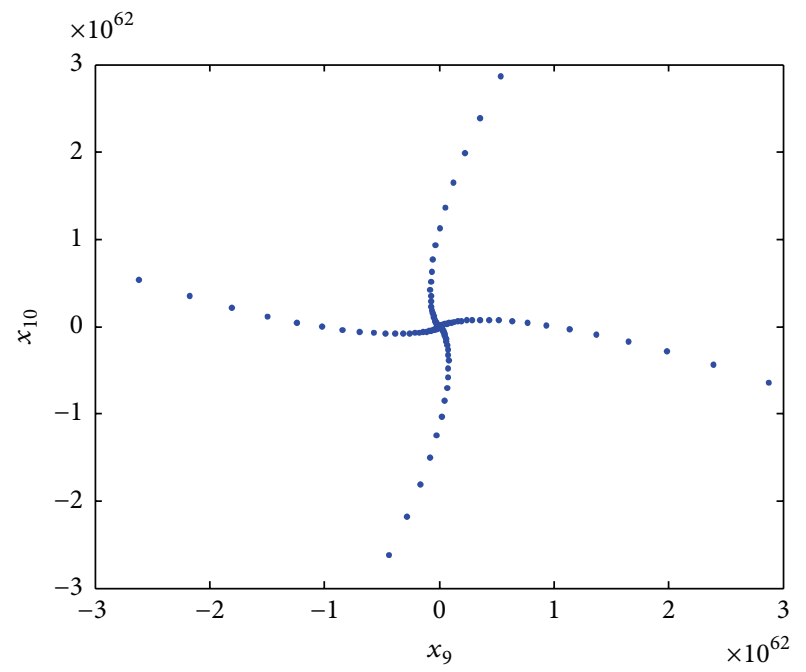

(b) Poincare maps

FIGURE 9: $\omega=262$ without random perturbation. 


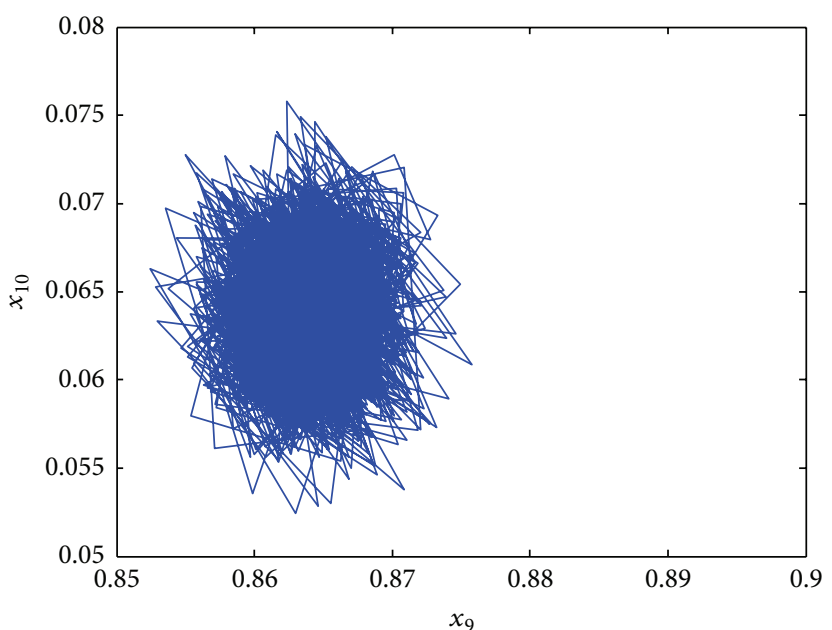

(a) Phase trajectories

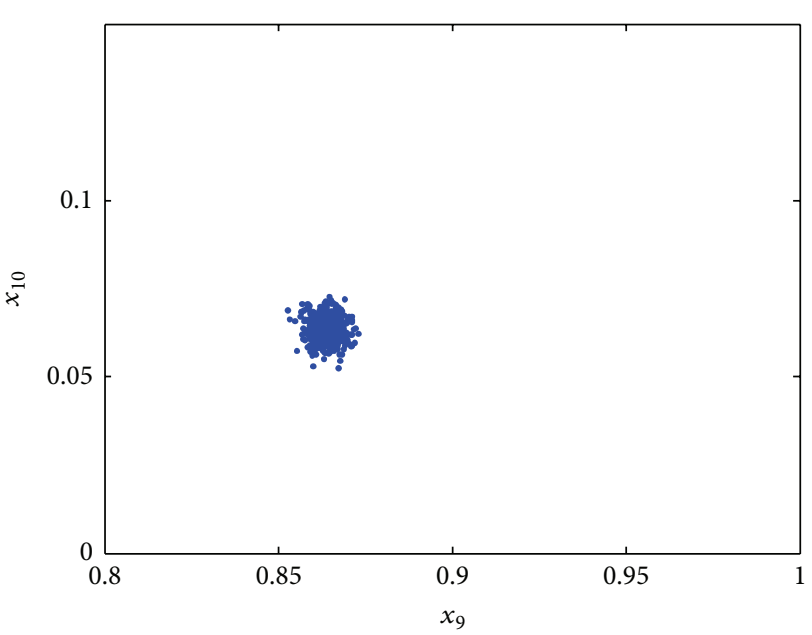

(b) Poincare maps

FIGURE 10: $\omega=110$ with random perturbation.

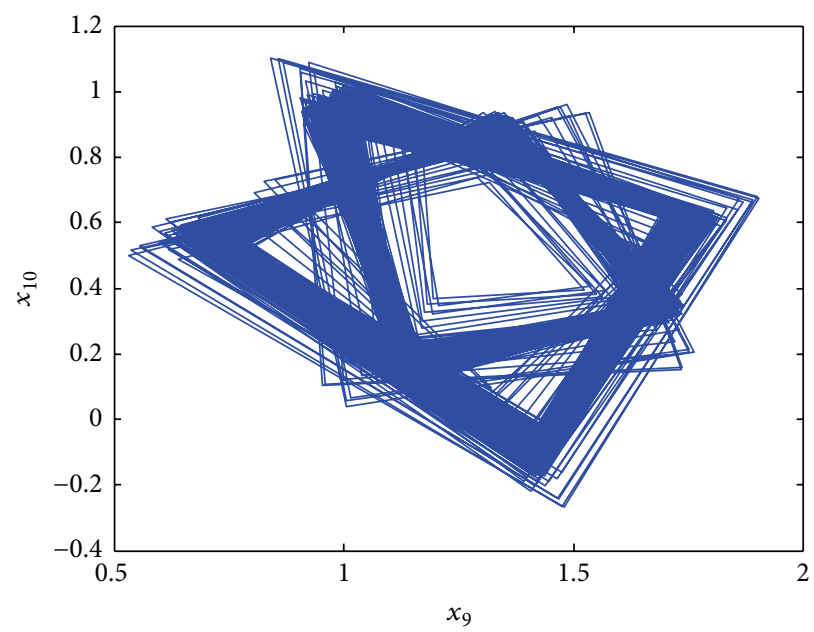

(a) Phase trajectories

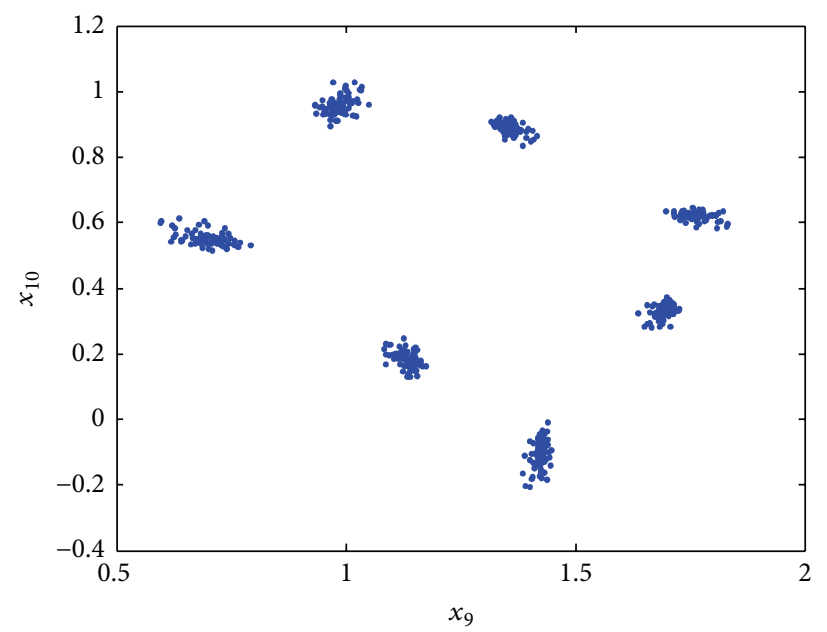

(b) Poincare maps

FIGURE 11: $\omega=175$ with random perturbation.

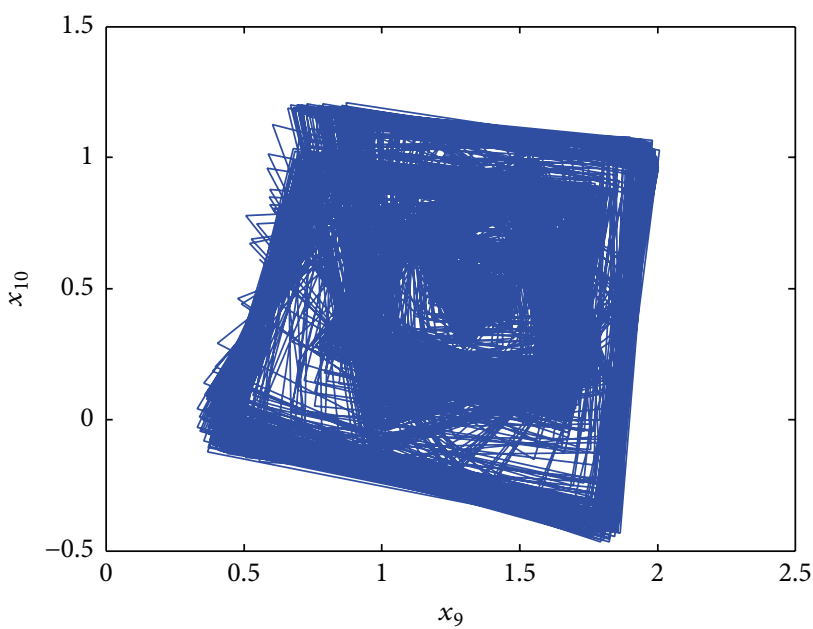

(a) Phase trajectories

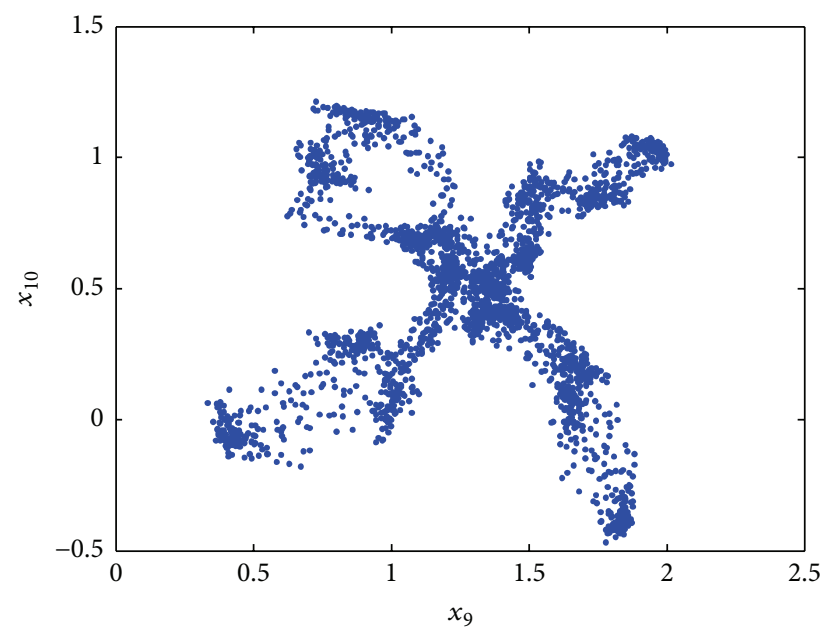

(b) Poincare maps

FIGURE 12: $\omega=180$ with random perturbation. 


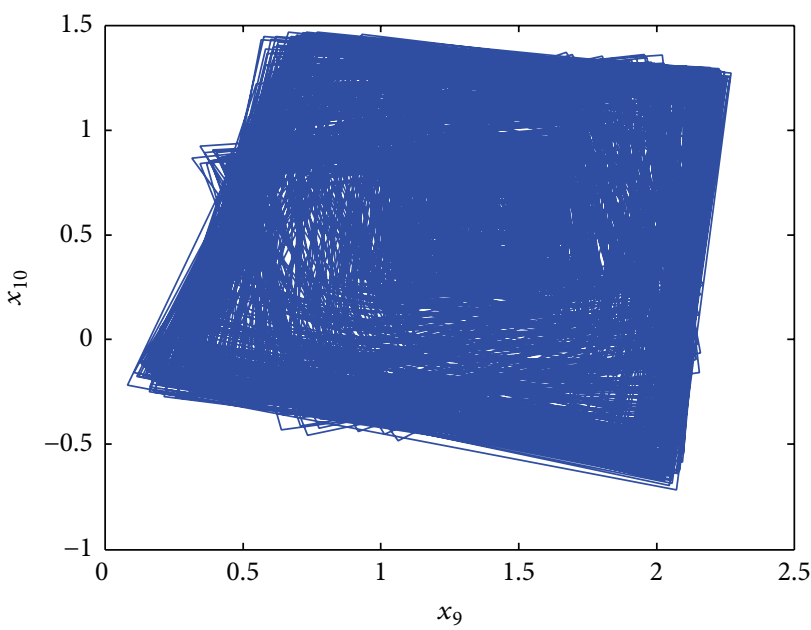

(a) Phase trajectories

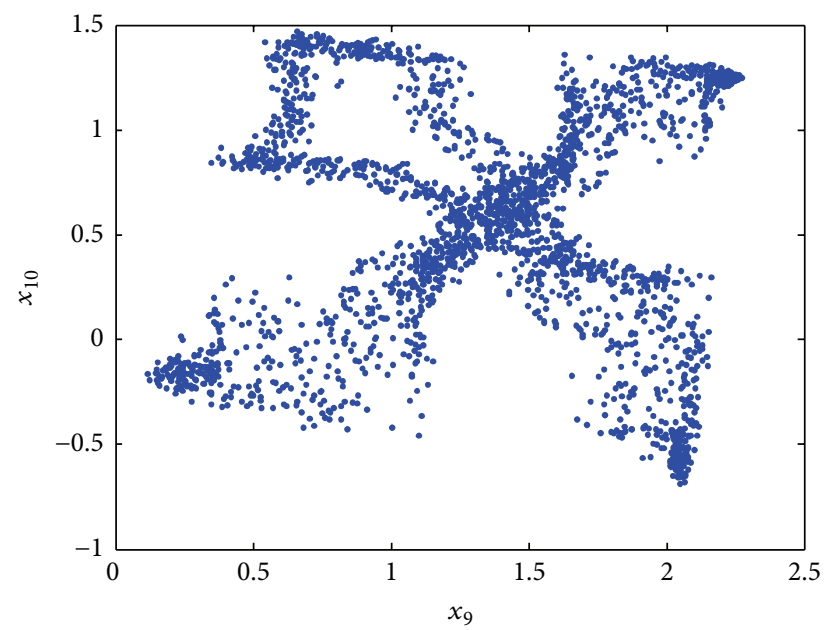

(b) Poincare maps

FIgURE 13: $\omega=200$ with random perturbation.

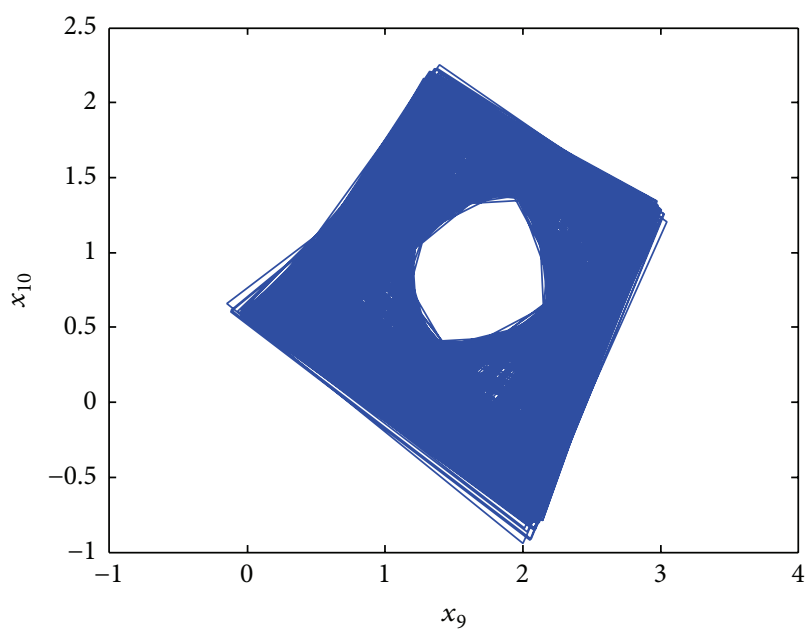

(a) Phase trajectories

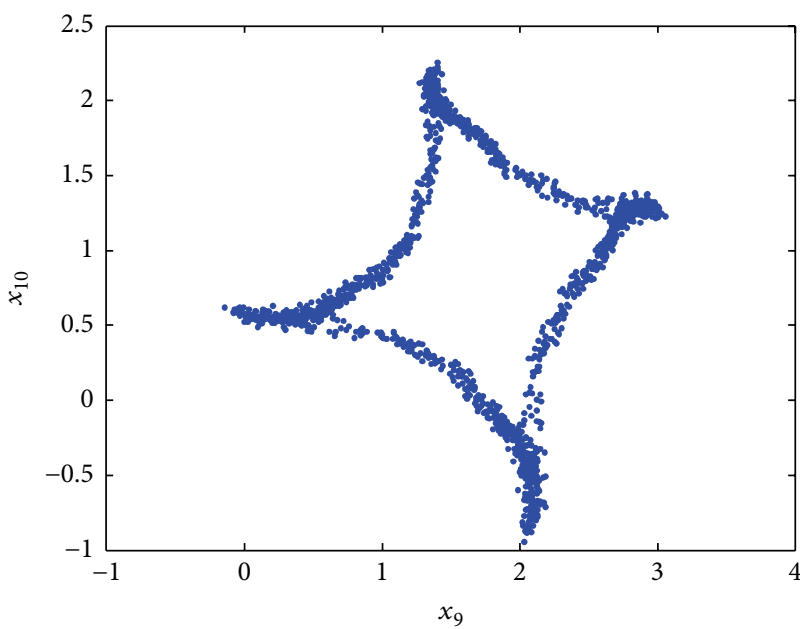

(b) Poincare maps

FIGURE 14: $\omega=240$ with random perturbation.

so forth are drawn in the following. Here, take $\omega=$ 175 , for instance. In the time-process diagrams in Figure 16, the amplitude without random perturbation is [ $-0.09510 .9517]$, while that with random perturbation is [-0.2586 1.0949]. The power spectrum in Figure 17 was depicted by a sampling frequency of 1000. And the Lyapunov exponents in Figure 18 were drawn by iteration time from 100 to 1000 . Neither of them changes much. In a nutshell, random perturbation does not change the system performance fundamentally.

However, if the value of $\delta_{F}^{*}$ fluctuates more violently, the dynamical property of the system will be changed. (1) When $\delta_{F}^{*} \sim N\left(0,0.01^{2}\right)$, the bifurcation of the system in Figure 19(a) is not clearly seen. (2) When $\delta_{F}^{*} \sim N\left(0,0.1^{2}\right)$, the bifurcation of the system disappears completely, and the system becomes uncontrollable in Figure 19(b).

\section{Conclusions}

This paper proposed a nonlinear dynamic model appropriate for the gear transmission system in a braiding machine. The influence of time-varying mesh stiffness, eccentric forces, multidisplacement functions of bearings, reactive forces of carriers, and so forth is fully considered in the mathematical model. The differential equations are similar to the Clifford system but contain more nonlinear terms. And the numerical results are accordant to those in [12]. Here, the Clifford system can be written as follows:

$$
\begin{aligned}
& x_{k+1}=\sin \left(a y_{k}\right)-z_{k} \cos \left(b x_{k}\right), \\
& y_{k+1}=z_{k} \sin \left(c y_{k}\right)-\cos \left(d x_{k}\right), \\
& z_{k+1}=e \sin \left(b x_{k}\right) .
\end{aligned}
$$

Based on the differential equations, bifurcation diagrams, Lyapunov exponents, Poincare maps, phase trajectories, and 


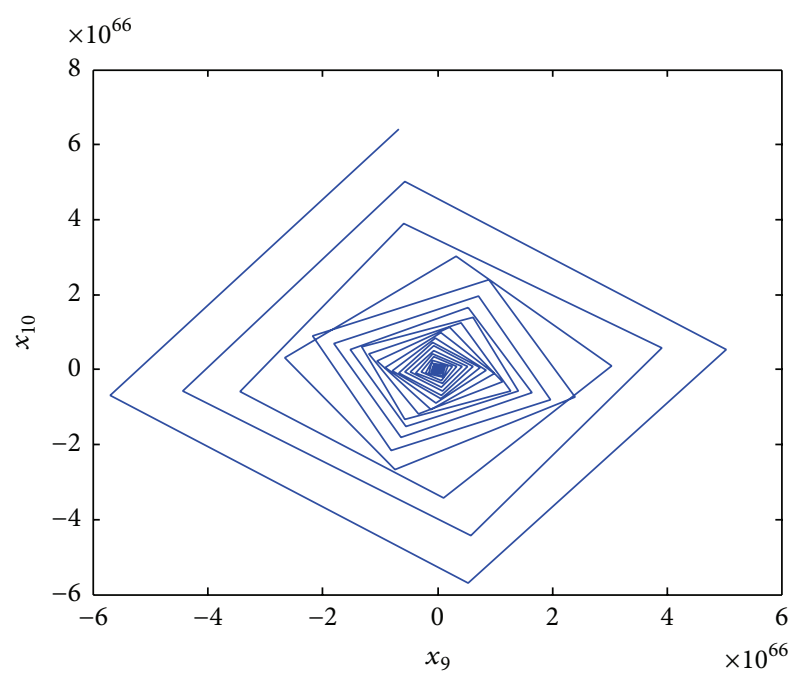

(a) Phase trajectories

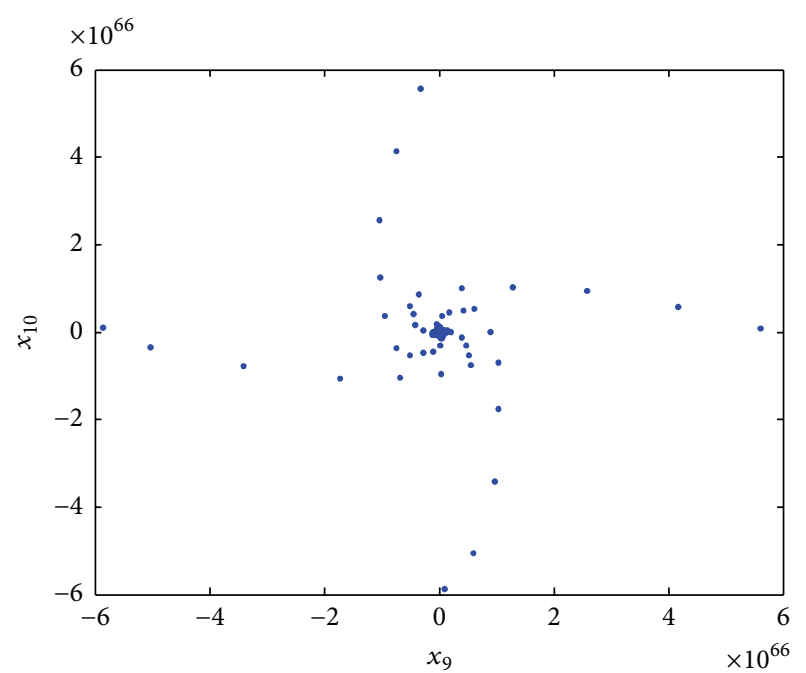

(b) Poincare maps

FIGURE 15: $\omega=262$ with random perturbation.

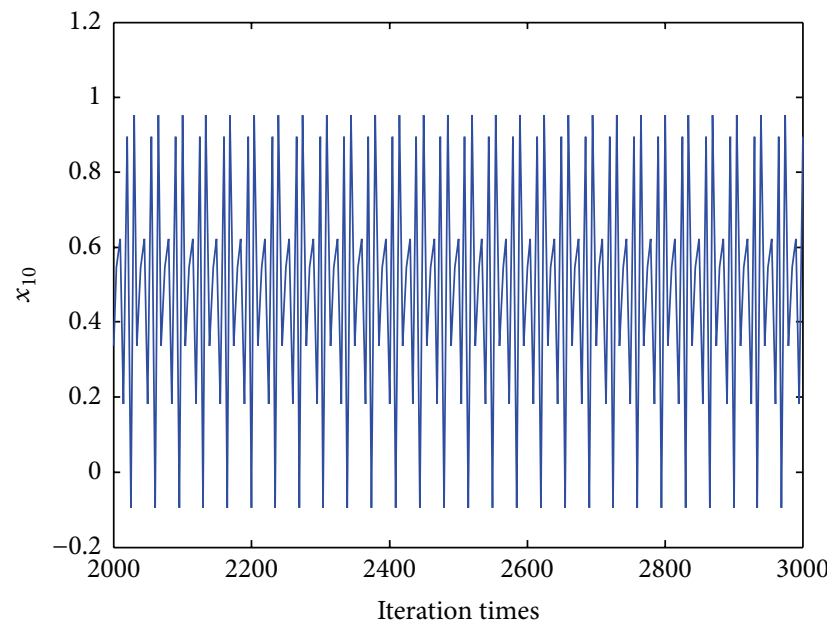

(a) Without random perturbation

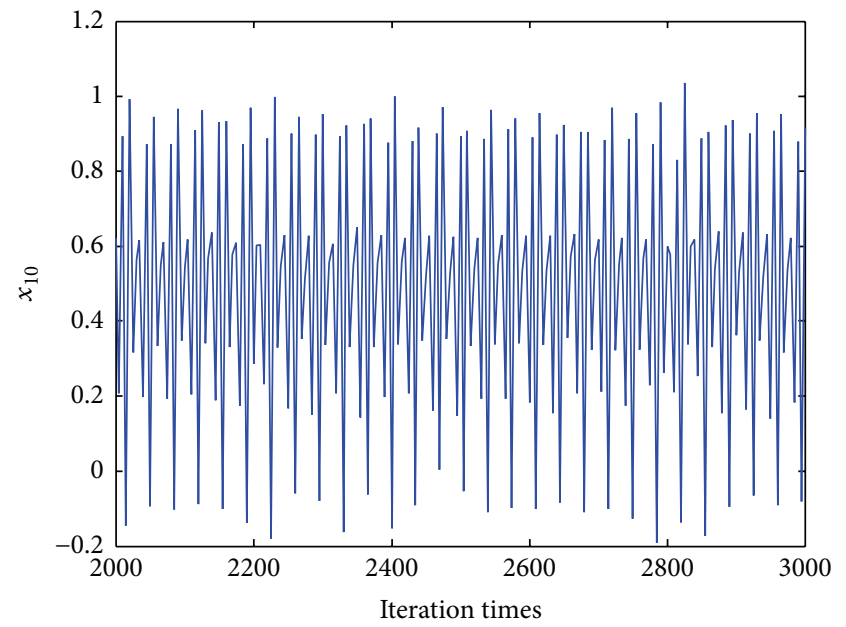

(b) With random perturbation

Figure 16: Time-process diagrams, $\omega=175$.

so forth are used to analyze the dynamic behavior of the system. In the bifurcation diagrams, it is shown that the bifurcation diagram of the system is similar to that of Clifford system, but more complex. As there are more nonlinear terms than those in Clifford system, the number of bifurcation points increases, and more periodic points can be seen. These causes the phenomenon of period doubling bifurcations cannot be distinguished significantly.

The results of numerical analysis have shown that, accompanied by the increasing of $\omega$, periodic bifurcations take place. When $\omega=180.06$, chaotic behavior can be observed. In other words, the dimensionless parameter $\omega$ ought to be controlled under 180.06, which is equal to a speed of $21125 \mathrm{r} / \mathrm{s}$. If the spur transferred from tension of yarns and security coefficient is taken into account, the safe speed in this braiding machine might be under about $250 \mathrm{r} / \mathrm{s}$, that is, $1.5 \times 10^{4} \mathrm{r} / \mathrm{min}$.
Although the theoretical speed is very high, it has no regard for random perturbation. If $\delta_{F}^{*} \sim N\left(0,0.1^{2}\right)$, the system of the braiding machine becomes uncontrollable. In other words, the dimensionless parameter $\delta_{F}^{*}$ ought to be controlled under an amplitude of 0.1 , which is equal to about $2.2 \mathrm{kN}$. Since there are hundreds of gears forming a long transmission chain, with many other excitations existing, it is very likely to reach the value. When this situation happens, the system might be out of order, which would result in the interruption of braiding process, or the breaking of yarns. Therefore, the values obtained above might be a reference during the design of this type of braiding machines.

\section{Conflict of Interests}

None of the authors have a conflict of interests. 


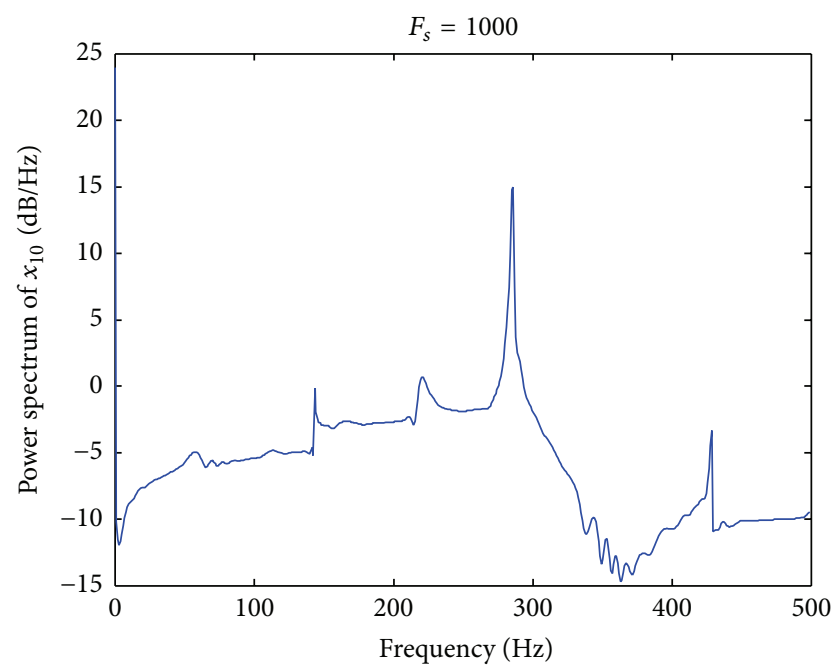

(a) Without random perturbation

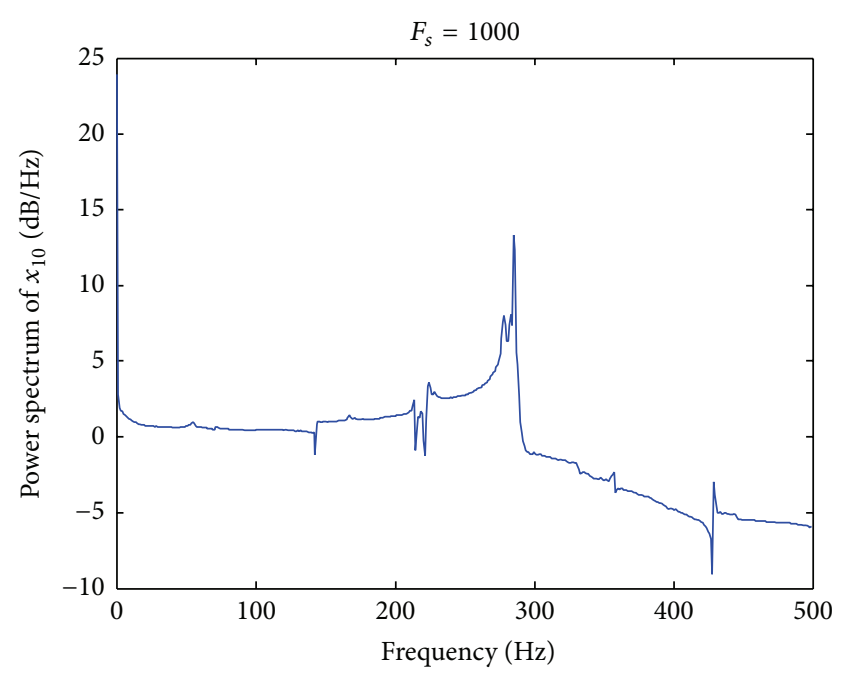

(b) With random perturbation

FIgURe 17: Power spectrum, $\omega=175$.

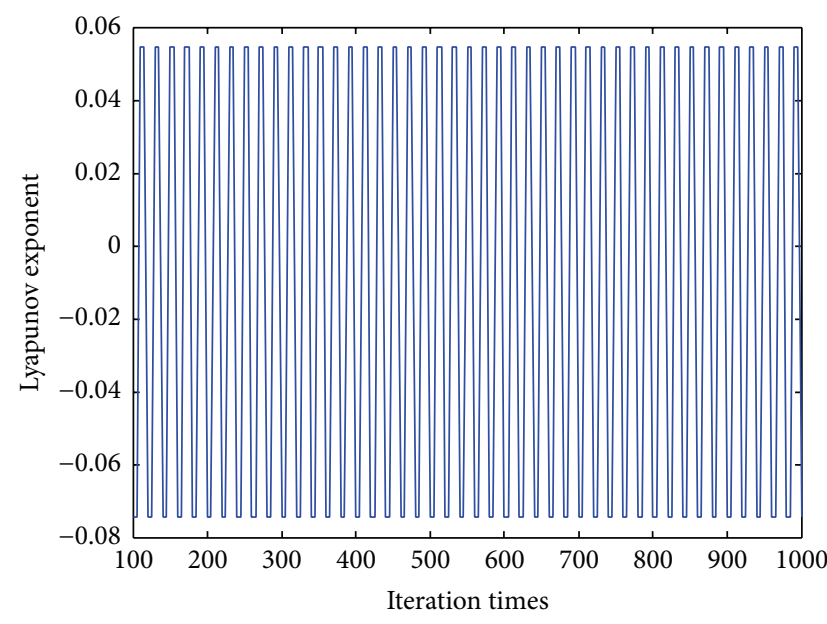

(a) Without random perturbation

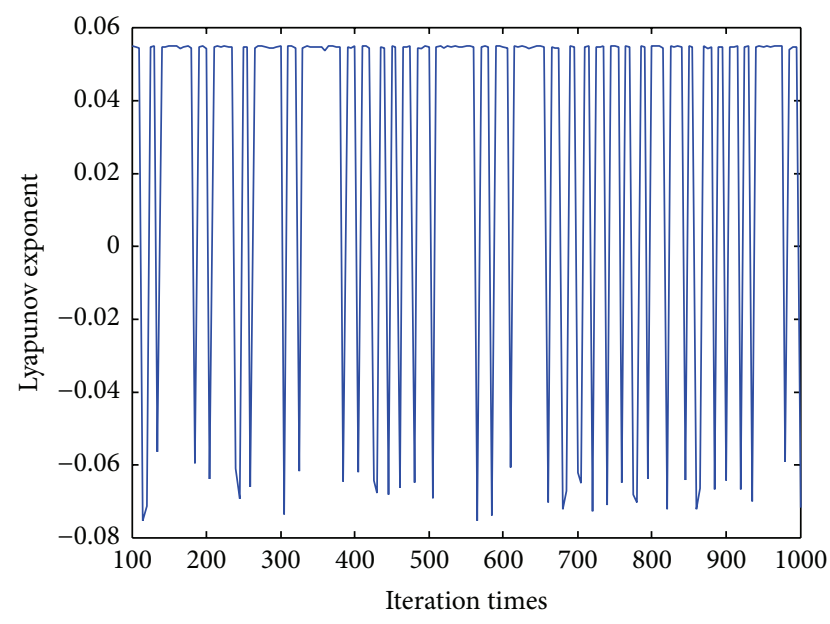

(b) With random perturbation

FIGURE 18: Lyapunov exponents, $\omega=175$.

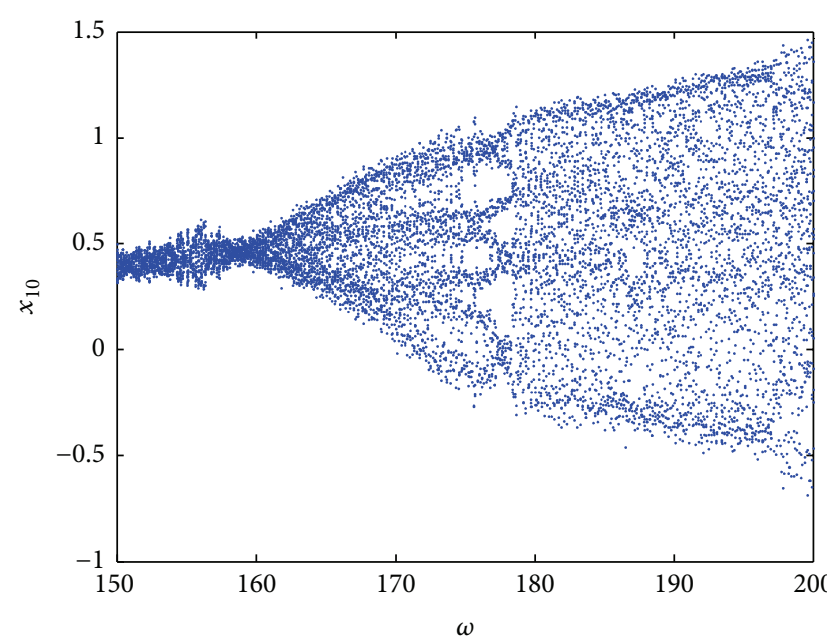

(a) $\delta_{F}^{*} \sim N\left(0,0.01^{2}\right)$

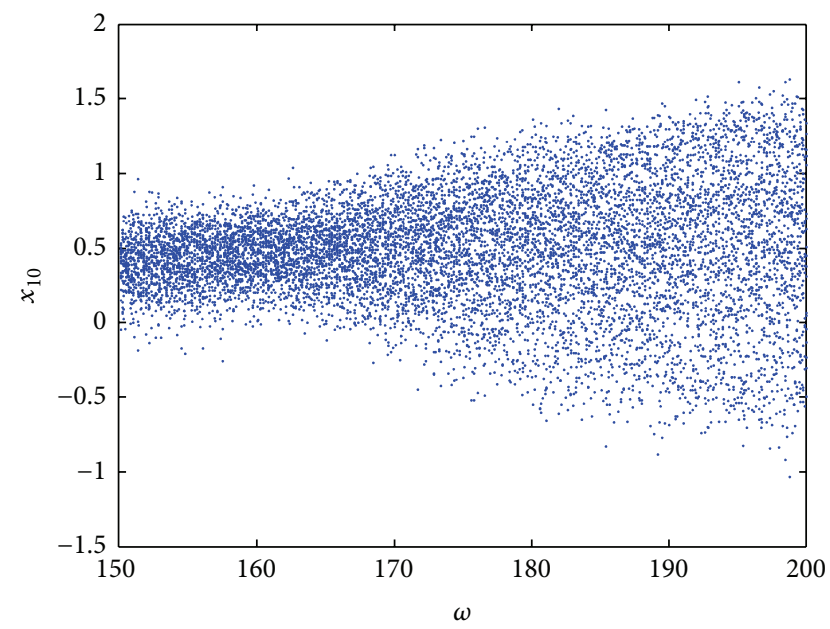

(b) $\delta_{F}^{*} \sim N\left(0,0.1^{2}\right)$

FIGURE 19: Bifurcation diagrams with random perturbation. 


\section{Acknowledgments}

This work was supported by the National Natural Science Foundation of China under Grant [51475091]; Program of Shanghai Leading Talent under Grant [20141032].

\section{References}

[1] Y. Kyosev, Braiding Technology for Textiles, Woodhead Publishing, Cambridge, UK, 2014.

[2] G. W. Blankenship and A. Kahraman, "Steady state forced response of a mechanical oscillator with combined parametric excitation and clearance type non-linearity," Journal of Sound and Vibration, vol. 185, no. 5, pp. 743-765, 1995.

[3] A. Kahraman and R. Singh, "Nonlinear dynamics of a spur gear pair," Journal of Sound and Vibration, vol. 142, no. 1, pp. 49-75, 1990.

[4] A. Kahraman and R. Singh, "Interactions between time-varying mesh stiffness and clearance non-linearities in a geared system," Journal of Sound and Vibration, vol. 146, no. 1, pp. 135-156, 1991.

[5] C.-W. Chang-Jian and S.-M. Chang, "Bifurcation and chaos analysis of spur gear pair with and without nonlinear suspension," Nonlinear Analysis: Real World Applications, vol. 12, no. 2, pp. 979-989, 2011.

[6] A. Raghothama and S. Narayanan, "Bifurcation and chaos in geared rotor bearing system by incremental harmonic balance method," Journal of Sound and Vibration, vol. 226, no. 3, pp. 469-492, 1999.

[7] J. Wang, J. H. Zheng, and A. B. Yang, "An analytical study of bifurcation and chaos in a spur gear pair with sliding friction," Procedia Engineering, vol. 31, pp. 563-570, 2012, Proceedings of the International Conference on Advances in Computational Modeling and Simulation.

[8] J. Wang, H. Wang, and L. Guo, "Chaotic vibration analysis of gear transmission system with stochastic disturbance," Journal of Vibration, Measurement \& Diagnosis, vol. 34, no. 6, pp. 10991104, 2014.

[9] L. Xiaolin, Non-linear vibration and chaotic contol of threedegree-freedom gear transmission system [M.S. thesis], Northwest Polytechnical University, Xian, China, 2003.

[10] H. Ma, R. Song, X. Pang, and B. Wen, "Time-varying mesh stiffness calculation of cracked spur gears," Engineering Failure Analysis, vol. 44, pp. 179-194, 2014.

[11] T. Eritenel and R. G. Parker, "An investigation of tooth mesh nonlinearity and partial contact loss in gear pairs using a lumped-parameter model," Mechanism and Machine Theory, vol. 56, pp. 28-51, 2012.

[12] T. L. D. Mbong, M. S. Siewe, and C. Tchawoua, "The effect of nonlinear damping on vibrational resonance and chaotic behavior of a beam fixed at its two ends and prestressed," Communications in Nonlinear Science and Numerical Simulation, vol. 22, no. 1-3, pp. 228-243, 2015. 


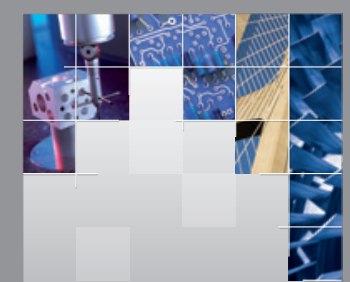

\section{Enfincering}
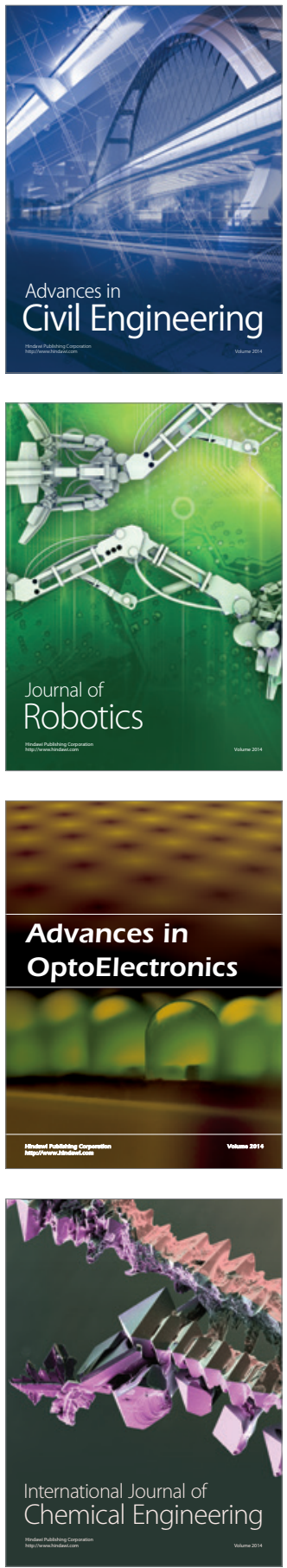

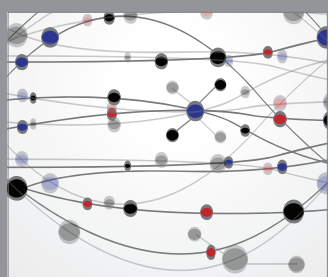

The Scientific World Journal

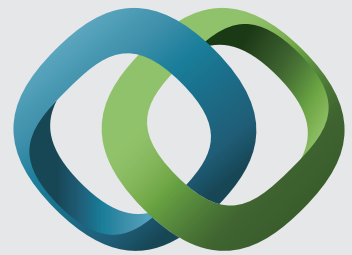

\section{Hindawi}

Submit your manuscripts at

http://www.hindawi.com
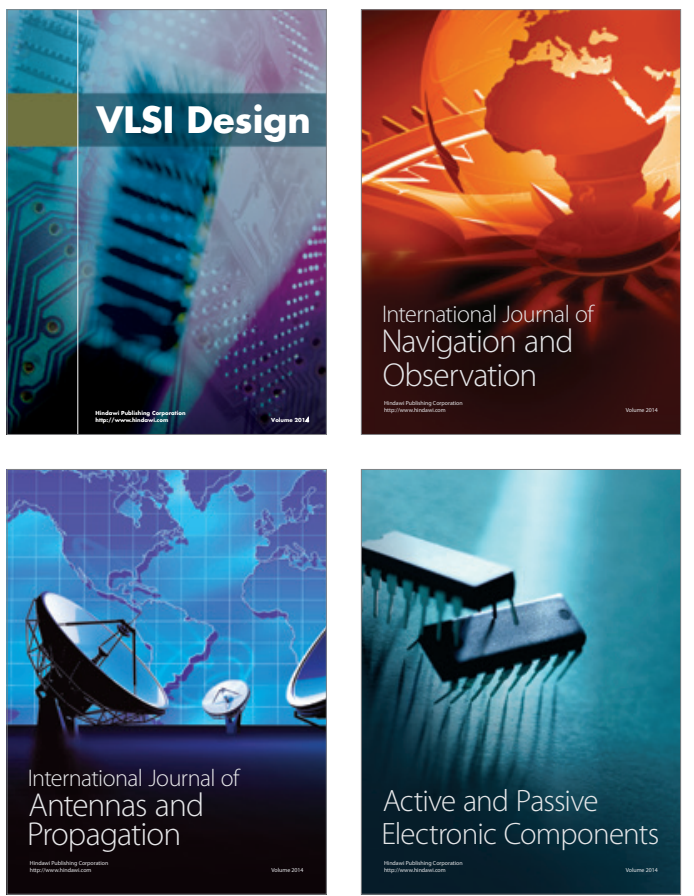
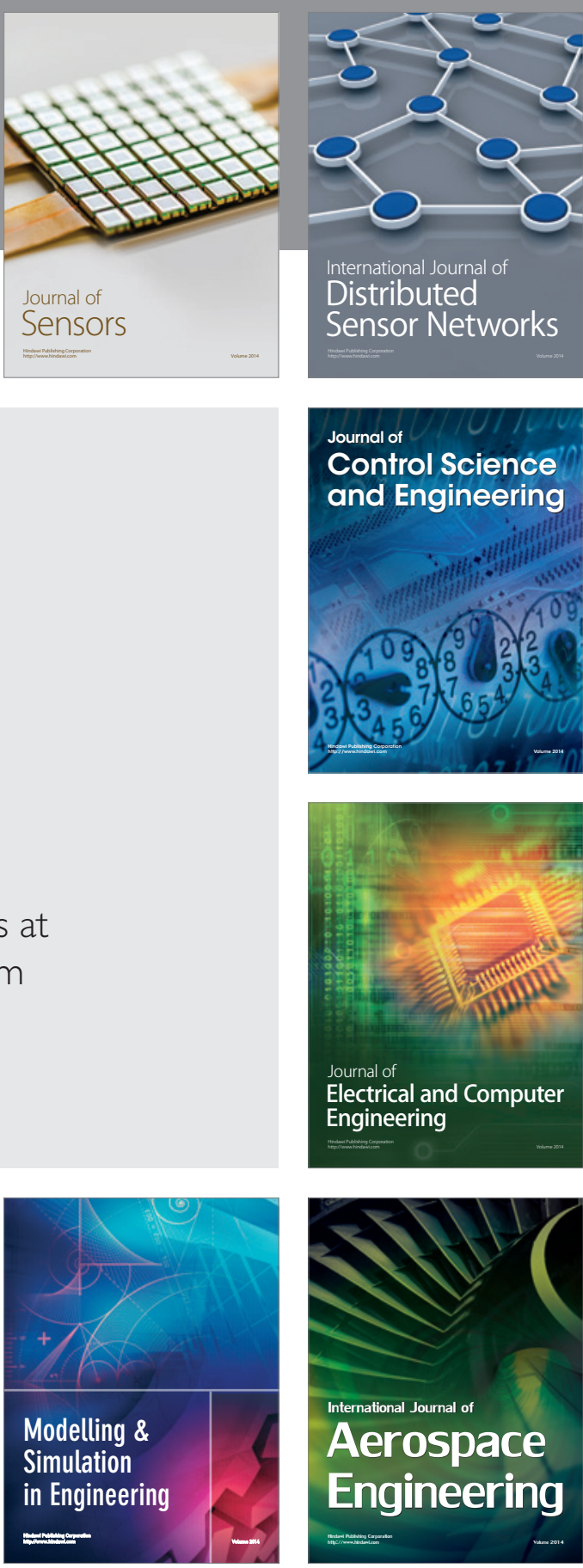

International Journal of

Distributed

Sensor Networks

Journal of

Control Science

and Engineering
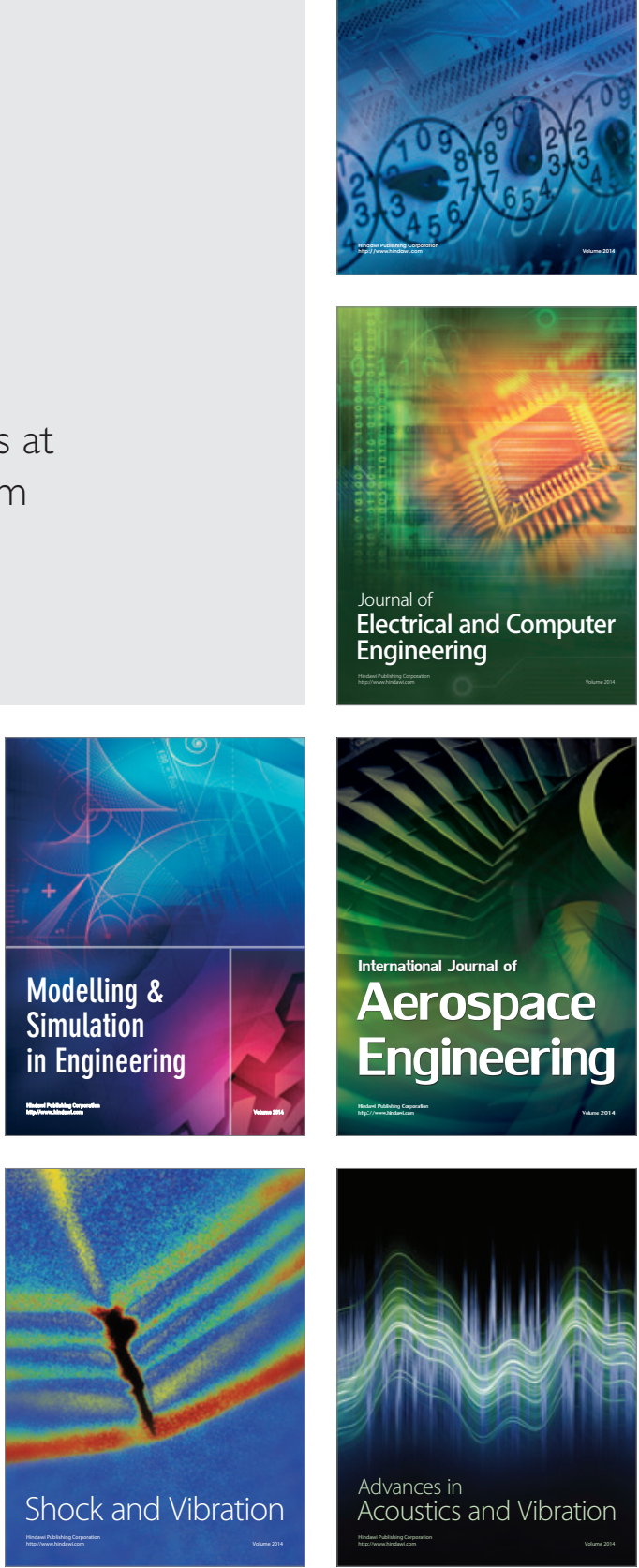